\title{
Barriers and facilitators to the integration of mental health services into primary healthcare: a qualitative study among Ugandan primary care providers using the COM-B framework
}

\author{
Edith K. Wakida ${ }^{1 *}$ (D), Celestino Obua ${ }^{2}$, Godfrey Z. Rukundo ${ }^{1}$, Samuel Maling ${ }^{1}$, Zohray M. Talib ${ }^{3,4}$ and
}

Elialilia S. Okello ${ }^{5}$

\begin{abstract}
Background: Uptake of clinical guideline recommendations into routine practice requires changes in attitudes and behaviors of the health care providers. The World Health Organization (WHO) has heavily invested in public health and health promotion globally by developing policy recommendations to guide clinical practice; however, clinical guidelines are often not applied. The success of the implementation of any guidelines depends on consideration of existing barriers and adequately addressing them. Therefore, exploring the context specific barriers and facilitators affecting the primary care providers (PCPs) in Mbarara district, Uganda may provide a practical way of addressing the identified barriers thus influence the PCPs action towards integration of mental healthcare services into PHC.

Methods: We adopted a theoretical model of behavior change; Capability, Opportunity and Motivation developed to understand behavior (COM-B). This was a cross-sectional study which involved using a semi-structured qualitative interview guide to conduct in-depth interviews with PCP's (clinical officers, nurses and midwives).

Results: Capability - inadequacy in knowledge about mental disorders; more comfortable managing patients with a mental problem diagnosis than making a new one; knowledge about mental health was gained during pre-service training; no senior cadre to consultations in mental health; and burdensome to consult the Uganda Clinical Guidelines (UCG). Opportunity - limited supply of hard copies of the UCG; guidelines not practical for local setting; did not regularly deal with clients having mental illness to foster routine usage of the UCG; no sensitization about the UCG to the intended users; and no cues at the health centers to remind the PCPs to use UCG. Motivation - did not feel self-reliant; not seen the UCG at their health facilities; lack of trained mental health specialists; conflicting priorities; and no regulatory measures to encourage screening for mental health.

Conclusions: Efforts to achieve successful integration of mental health services into PHC need to fit in the context of the implementers; thus the need to adapt the UCG into local context, have cues to enforce implementation, and optimize the available expertize (mental healthcare providers) in the process.
\end{abstract}

Keywords: Integration of mental health services, Primary care providers, Uganda clinical guidelines, Capability, Opportunity and motivation

\footnotetext{
* Correspondence: ediwakida@must.ac.ug

${ }^{1}$ Department of Psychiatry, Mbarara University of Science and Technology, P.

O. Box 1410, Mbarara, Uganda

Full list of author information is available at the end of the article
}

(c) The Author(s). 2018 Open Access This article is distributed under the terms of the Creative Commons Attribution 4.0 International License (http://creativecommons.org/licenses/by/4.0/), which permits unrestricted use, distribution, and reproduction in any medium, provided you give appropriate credit to the original author(s) and the source, provide a link to the Creative Commons license, and indicate if changes were made. The Creative Commons Public Domain Dedication waiver (http://creativecommons.org/publicdomain/zero/1.0/) applies to the data made available in this article, unless otherwise stated. 


\section{Background}

Uptake of clinical guideline recommendations into routine practice requires changes in attitudes and behaviors of the health care providers as well as structural changes in their work environment [1,2]. Evidence suggests that in order to promote adherence to guideline recommendations, there is need for structural adaptation to specific settings and target populations at different levels [3, 4].

The World Health Organization (WHO) has heavily invested in public health and health promotion globally by developing policy recommendations to guide clinical practice [5], and one such investment is promotion of mental health services into primary healthcare (PHC). Through the Alma-Ata declaration [6] PHC was adapted as the preferred method for providing a comprehensive, universal, equitable and affordable healthcare that could reduce stigma, improve access to care, reduce chronicity of mental illness and improve social integration [7-9]. In the Alma-Ata declaration, it was recommended that countries transform their mental health services to: i) promote self-care, ii) build informal community care services, iii) build community mental health services, iv) develop mental health services in general hospitals, and v) limit reliance on psychiatric hospitals [10]. In addition, WHO issued recommendations to guide the integration process at PHC level [9] because it was seen as the most viable way of closing the treatment gap and ensuring that people get the mental healthcare services they need [11]. Integration of mental health services into PHC has been embraced by various countries and in different forms [12-15] including a) training primary care providers (PCPs) in identifying mental health problems; b) PCPs assessing for mental illnesses during medical standard of care; c) PCPs/ Community Health Workers (CHWs) and health care managers working together to address mental health related illnesses; and d) availing psychotropic medications to PHC centers [16]. Despite all these efforts, PHC for mental health has not been realized in most countries of the world; it is estimated that the treatment gap is widest among people with severe mental disorders in least resourced countries [11, 17-19].

In Uganda, mental health problems are recognized as a public health challenge contributing $13 \%$ to the national disease burden [20-22], and as such the Ministry of Health made health reforms to adopt the WHO recommendations of integrating mental health services into PHC $[9,11]$, these included: decentralizing the health delivery structures to the lowest level of care [11, 23]; formulation of the Uganda Minimum Health Care Package (UMHCP) with mental health as a key component [23, 24]; developing the Uganda Clinical Guidelines (UCG) on the management of common disorders [25, 26]; and training PCPs to identify and manage mental health problems, and refer complicated cases to higher levels of care [11]. However, there is still a gap in provision of mental health services at PHC inspite of all these reforms and the fact that research evidence suggests that mental healthcare can be delivered effectively in PHC settings $[11,17,27,28]$.

Furthermore, literature shows that clinical guidelines are often not applied and that the success of their implementation depends on consideration of existing barriers and adequately addressing them [29]. Interventions directed towards specific barriers are more effective than those that are not, thus the need for a planned introduction of tailored guidelines that are culturally sensitive to the targeted population [4, 30]. This study was developed following our earlier study about the need to conduct context specific studies to identify the barriers and facilitators the PCPs face in integrating mental health services into PHC; Wakida EK, Talib ZM, Akena D, Okello ES, Kinengyere A, Obua C. Barriers and facilitators to the integration of mental health services into primary health care: A systematic review [Forthcoming].

Using a case study approach $[31,32]$ and the Capability, Opportunity and Motivation framework for understanding behavior [33] the aim of this study was to explore the context specific factors affecting the ability of PCPs in rural Mbarara district to integrate mental health services into PHC. Understanding these factors may provide a practical way of addressing the identified barriers thus influence the PCPs action towards integration of mental healthcare services into PHC. In addition, conclusions from the findings may make it possible to design a relevant intervention tailored to the context of the PCPs in order to promote better implementation of the policy option. In the Ugandan healthcare system, PCPs, including medical officers, clinical officers, nursing officers and midwives are directly involved in assessing and managing mental health problems.

\section{Theoretical framework}

In this study we adopted a theoretical model of behavior change; Capability, Opportunity and Motivation developed to understand behavior (COM-B) [33-35]. The theory postulates that in order to change behavior, there should be an interaction between one or more of 'capability' to perform the behavior and/or 'opportunity' and 'motivation' to carry out the behavior (Fig. 1). According to the proponents of this theory [33], Capability is the individual's psychological and physical capacity to engage in an activity (knowledge and skills); Motivation are brain processes that direct behavior, (goals, conscious decision-making, habitual processes, emotional responding, and analytical decision-making); while Opportunity are the factors (physical or social environment) that make the behavior possible or prompt it. In Fig. 1, motivation is influenced by both capability and opportunity, 


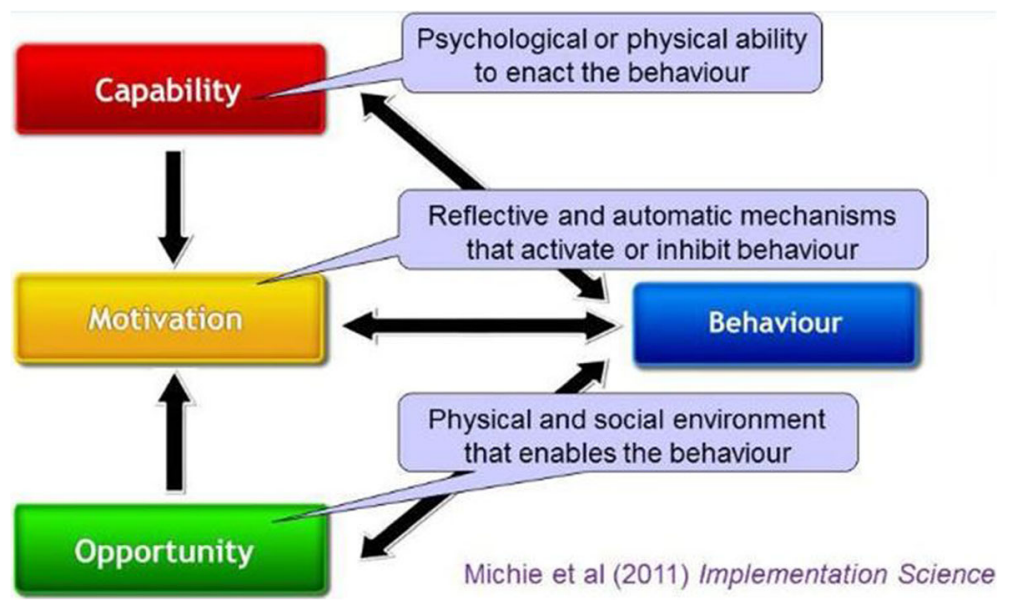

Fig. 1 COM-B Framework for understanding behavior

therefore enacting a behavior can alter capability, motivation, and opportunity. Emergent barriers and facilitators were identified using this framework.

\section{Methods}

\section{Study design}

This was a qualitative study that used thematic analysis to examine the barriers and facilitators to the integration of mental health services into PHC by PCPs in rural Mbarara district, Uganda. We used the a semi-structured interview guide to enable us understand the phenomena in its natural environment $[32,36]$. The study was designed by EW in consultation with ESO, ZT, and CO; questions were developed based on the COM-B framework to enable us pick the emergent factors influencing the PCPs behavior towards the policy option. The interview guide was pilot tested at a HC that was not included in the study, and revisions made based on lessons learned.

\section{Study setting}

The study was conducted in Mbarara district approximately $270 \mathrm{~km}$ (170 miles), by road, southwest of the capital city, Kampala. Mbarara is the administrative capital of southwestern Uganda and it boarders Ibanda and Kiruhura Districts to the north, Kiruhura and Isingiro Districts to the east, Isingiro and Ntungamo Districts to the south, and Sheema District to the west [37]. Demographically, Mbarara district lies between coordinates 00 $36 \mathrm{~S}, 3036 \mathrm{E}$ and covers an area of $1846.4 \mathrm{~km}^{2}$ with a population of 472,625 of which $242,547(51.3 \%)$ are females [37].

In Uganda provision of mental health services begins at health center III (sub-county level) and with subsequent referrals to HC IV (county level), district hospitals, regional referral hospitals and finally to the national referral hospital [26, 38]. Each health facility level (except
HC III) is expected to have general doctors (medical officers), clinical officers (Diploma level Medical Assistants), nurses and midwives, and psychiatric nurses. The HC IIIs do not have general doctors but have all the other cadres of service providers. Mbarara district has $13 \mathrm{HC}$ IIIs and four HC IV; provision of health services is spearheaded by the district health department responsible for curative and preventive healthcare [39]. All the $\mathrm{HC}$, as the primary health facilities, that were included in this study are located in rural Mbarara district.

\section{Participant's recruitment}

Participants who took part in the study included clinical officers, nurses and midwives from six health centers (III and IV); doctors were not part of the study because the HCs either did not have general doctors in the establishment (all HC III), did not provide consent to participate or were not available at the time of the study (on official assignment off station). There are four HC IV in Mbarara district and each has only one Medical doctor.

The sampling frame for our study was $17 \mathrm{HCs}(13 \mathrm{HC}$ III and four HC IV). Each category (HC III and HC IV) was evaluated for inclusion based on whether they were a government facility, had PCPs' who directly assessed patients, and not neighboring a similar health facility (including privately owned), or not located near a hospital. We randomized the facilities by strata (HC III and HC IV) to obtain an equal number of HCs per cluster.

While we proposed to consider age, gender, occupation and seniority/experience when selecting participants, we found on the ground that the health facilities had a smaller health workforce than we had anticipated (about five to eight per $\mathrm{HC}$ ), thus we recruited all PCPs we found at the facilities and only interviewed those who provided signed consent. A total of 20 in-depth interviews were conducted, 12 participants were from $\mathrm{HC}$ 
IV and eight from HC III. In our view, 20 is sufficient to exhaustively generate information, and by the time we interviewed the last participant, we were eliciting similar responses with no new information being identified. In terms of gender we had more females $(n=18)$ than males $(n=2)$. There were no significant age differences between the study participants with ages ranging between 30 and 49 years at the time of the study (Table 1). In terms of position or health cadre's levels, we found 10 nurses, four midwives, two psychiatric nurses, and four Clinical Officers in the health facilities. Views of all the participants were included in the analysis and contribute to the conclusions in our study.

\section{Procedure}

In-depth interviews were conducted by the lead author (EW) and two trained research assistants (MN and CK) between November 2017 and April 2018. Each interview lasted approximately $60 \mathrm{~min}$ and was audio recorded. All interviews were conducted in English the national official language, and backed by field notes.

\section{Data collection and tools}

A semi-structured interview guide was developed by $\mathrm{EW}$ in consultation with $\mathrm{ESO}, \mathrm{CO}$, and $\mathrm{ZT}$; questions were developed based on the capability, opportunity and motivation framework for understanding behavior
(Additional file 1). They focused on the use of existing Uganda Clinical Guidelines (UCG) when assessing for mental health problems. The UCG helps clinicians by presenting updated, practical, and useful information on the diagnosis and management of common conditions in Uganda. The interview guide was pilot tested at a health facility that was not included in the study.

\section{Data management and analysis}

Data were transcribed verbatim by the research assistants, and checked by EW against the audio recordings for correctness of information before proceeding to the next set of interviews. Using the first set of transcripts, clarification was sought from ESO, CO, and ZT, to ensure that the questions were being asked and responded to in the correct way and would answer the research question. Data were thematically analyzed [40] with the help of a qualitative software Atlas.ti version 7 [41]. ESO and EW independently read through transcripts and developed codes in accordance with the COM-B domains capability, opportunity and motivation and the initial coding done by EW. The coding process was discussed with $\mathrm{CO}$ a senior researcher, and $\mathrm{ZT}$ a health policy expert. There was an iterative process during the coding to agree on which responses were either barriers or facilitators and which ones belonged to the capability, opportunity and motivation domains.

Table 1 Summary of participants characteristics

\begin{tabular}{|c|c|c|c|c|}
\hline Participant & Age & Gender & Health cadre & Level of education \\
\hline $\mathrm{P} 1$ & 38 & Female & Nursing officer & Diploma in Nursing and Midwifery \\
\hline P2 & 41 & Male & Clinical officer & Degree in Public Health \\
\hline P3 & 31 & Female & Enrolled nurse & Certificate in Nursing \\
\hline P4 & 32 & Female & Psychiatric nurse & Certificate in Mental Health Nursing \\
\hline P5 & 32 & Female & Nursing officer & Diploma in Nursing \\
\hline P6 & 45 & Female & Senior Nursing officer & Diploma in Nursing \& Health service Management \\
\hline P7 & 39 & Female & Midwife & Certificate in Midwifery \\
\hline P8 & 49 & Female & Enrolled nurse & Certificate in Nursing \\
\hline P9 & 32 & Male & Clinical officer & Diploma in Clinical Medicine \\
\hline P10 & 30 & Female & Midwife & Certificate in Midwifery \\
\hline P11 & 35 & Female & Enrolled nurse & Certificate in Nursing \\
\hline P12 & 49 & Female & Nursing officer & Diploma in Nursing and Midwifery \\
\hline P13 & 31 & Female & Psychiatric nurse & Diploma in Mental Health Nursing \\
\hline P14 & 38 & Female & Senior Nursing officer & Diploma in Nursing \\
\hline P15 & 32 & Female & Midwife & Certificate in Midwifery \\
\hline P16 & 32 & Female & Clinical officer & Degree in Public Health \\
\hline P17 & 38 & Female & Clinical officer & Diploma in Clinical Medicine \\
\hline P18 & 30 & Female & Nursing officer & Diploma in Nursing \\
\hline P19 & 47 & Female & Midwife & Certificate in Midwifery \\
\hline P20 & 30 & Female & Enrolled nurse & Certificate in Nursing \\
\hline
\end{tabular}




\section{Ethical considerations}

The study was approved by the Gulu University Research Ethics committee (GUREC), and the Uganda National Council of Science and Technology (UNCST). Permission to conduct interviews in the Health Centers in Mbarara was obtained from the District Health Officer. All participants provided written informed consent was obtained before each in-depth interview. Privacy of the participants was ensured by not including identifiable information in addition to conducting the interviews in private space. We respected individual autonomy to participate in the study by not including those who declined to participate, all who consented to participate were informed about their freedom to withdraw from the study at any time; no participant withdrew from the study. All the audio recorded material and transcripts were kept by to the lead author (EW).

\section{Results}

We structured the identified barriers and facilitators (Fig. 2) around three domains of behavior change: i) Capability which is the individual's psychological and physical capacity to engage in the activity concerned, ii) Opportunity - all the factors that lie outside the individual that make the behavior possible or prompt it, and iii) Motivation - all those brain processes that energize and direct behavior, not just goals and conscious decision-making. Although our participants across the $\mathrm{HC}$ comprised of 14 nurses with different levels of training and seniority (2 Senior Nursing Officers and 4 Nursing Officers, 4 Enrolled Nurses, 2 Psychiatric Nurses), 4 Midwives, and 4 Clinical Officers; the results presented in this section generally cut across the participants regardless of health cadre and facility level (HC III or IV). This could be explained by the fact that nearly all PCPs at different levels performed the same tasks of assessing patients and multitasking in addition to their other roles.

\section{Capability}

The ability of PCPs to integrate mental health services into PHC was explored; majority of the participants expressed inadequacy in knowledge about the various mental disorders, causes and management. This was attributed to the fact that during their training, they studied purely to pass exams. Patients with mental illness are therefore treated as any other patients without paying attention to their mental condition.

I can make a diagnosis of mental illness, but because they are very many types, I may not be able to differentiate them if the signs are not clear...when we were in school (medical) we read to pass exams and then we come here and we are

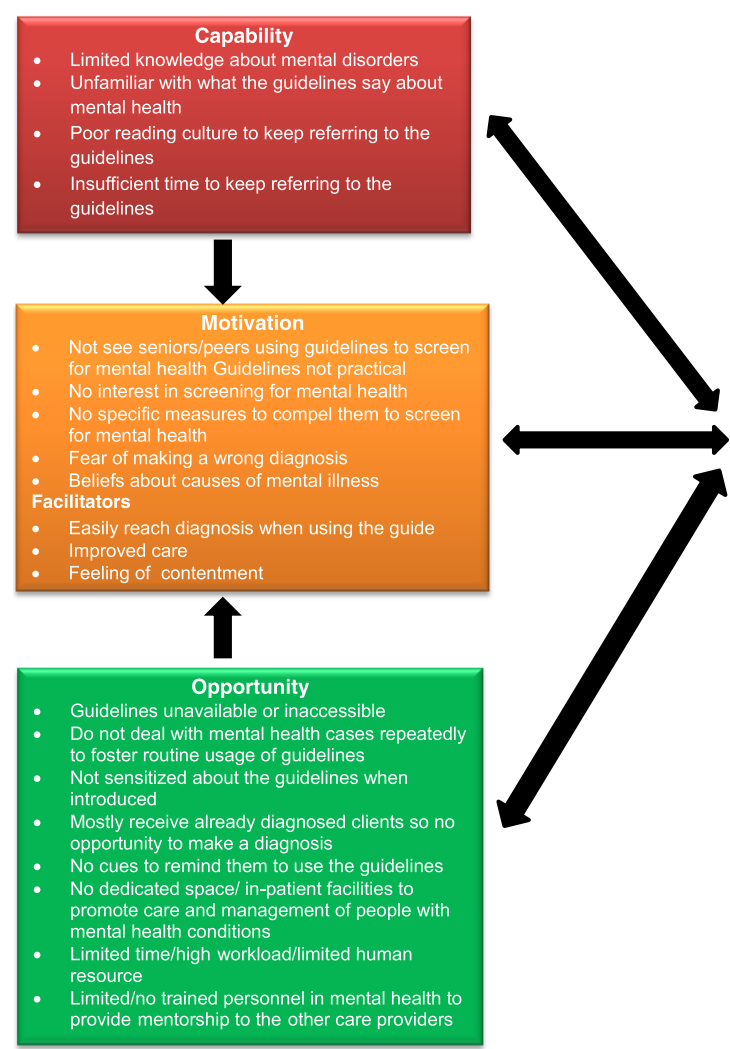

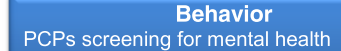

PCPs screening for mental heaith conditions using the Uganda Clinical Guidelines

Fig. 2 Emergent barriers and facilitators 
supposed to diagnose for mental illness; but that was not the focus so we just treat (mental health problems) like any other minor illness (Clinical Officer Health Facility (HF) 2).

Participants seemed more comfortable managing patients with a diagnosis of mental health problems than making a new one. We found that the health centers received patients who had received a diagnosis directly from Mbarara Regional Referral Hospital and were coming for medicine refills; thus preferred to handle such cases.

As I told you, for us we do the basics then the rest refer. I think even the structures we have cannot allow us to handle those people from here completely. We don't have isolation rooms for those people that's why I say we cannot manage them unless they are treated from the other side and when they calm down, we can continue with their treatment (Nursing Officer HF 1).

In addition, the knowledge about mental health that the participants had was gained during pre-service training; we found that since they started clinical practice, they had not received in-service training in the mental health area.

I can say that the knowledge I have on mental illness I got during my training. We do not have in-service training about mental health or the updates; at least if we are updated and we have that knowledge, we can handle (Nursing Officer HF 2).

There was no senior cadre to seek consultations when not sure mental condition hence referrals of clients who should have otherwise been managed at the lower level. The participants indicated that they preferred management of patients already on treatment.

I do not think am very comfortable making a diagnosis of mental health problems...there is no colleague to ask if I find something confusing, so I refer to Mbarara Regional Referral Hospital because I know there are specialists there (Clinical Officer HF 1).

When asked if they knew about the UCG and how helpful they could be when assessing for mental health problems, we found that the PCPs were aware about the UCG but found it burdensome to consult them unless they were totally in a fix as illustrated below by one participant.

To tell the truth it is not my culture to look through those guidelines (UCG) unless I am really cornered with a mother having signs of mental (Midwife HF 6).

\section{Opportunity}

We found that not all the PCPs had the opportunity to access the UCG because of the limited number of hard copies supplied to the respective health centers to be shared by all. This posed a challenge of knowing who last used the available copy and where it was kept in case they needed to refer to them.

When you get a client with signs of mental illness, you have to look for the guidelines (UCG), however, this process delays the patient or management of their condition; and it's a challenge when you also have to look for the dose, calculate, and then think about the side effects of the drug (Midwife HF 3).

The participants who had gained access to the UCG alluded to the fact that they were not practical for use in the local setting and needed to be summarized in a chart form to help them make the correct assessment of patients they suspect could be presenting with signs of mental illness. They noted that the UCG were very detailed and that the content about mental health was hidden and not quickly accessed when required.

Do you see how they have done for HIV care summarizing information on the charts and pinning everywhere...do the same for mental health. I don't think I can leave a patient in front of me that I am looking for the guidelines (UCG) to read, they need a lot of time to search for information (Clinical Officer HF 6).

The PCPs added that they did not repeatedly deal with clients having mental illness to foster routine usage of the UCG, thus found it bothersome to make reference each time the need arose. The health centers largely receive clients already diagnosed with mental health problems, and only need refills of their medication; there is limited opportunity for the PCPs to make a new diagnosis of mental health problems.

If we were seeing patients with mental illness on a daily basis with the same complaint, we would get used to the guidelines (UCG), but the patients (with mental health related problems) are not common...we mostly receive those with a diagnosis for refills so it is a challenge when we have to go and read the guidelines because we are not used to those patients (Midwife HF 4).

Exacerbating the PCPs challenges of integrating mental health services into $\mathrm{PHC}$ is that there was neither sensitization about the UCG to the intended users nor provision of sufficient copies to promote selfsensitization. 
We were just told that there are new guidelines to follow...they did not tell us how to use them and also did not give us personal copies. If at lease each one has a copy on their table, then maybe we can read them (Enrolled Nurse HF 1).

There were no cues at the health centers to remind the PCPs to use UCG when assessing for mental health problems. The PCPs felt that mental health was not as important as other disease where a lot of attention and resources were directed. Some of the health centers had donor funded projects with a set agenda and necessary resources to achieve the aims; this was not known to some participants thus causing them to think that mental healthcare is neglected and therefore not important.

Nobody asks us about people with mental problems the way they follow up with other programs like HIV, TB, malaria and immunization...I think if the ministry wants us to comply with mental health, let them support it like the other programs (Nursing Officer HF 4).

The other barrier related to opportunity was that the PCPs who were trained as mental healthcare providers and posted to the various health centers in rural Uganda were not necessarily practicing in their area of training. This caused the PCPs to feel deprived of time to do what they would be happy doing. The mental health integration policy requires all healthcare providers at $\mathrm{PHC}$ (lower levels of healthcare) to assess for mental health problems within routine practice. This in a way defeats the purpose of specialized training to practice at lower levels of healthcare where ones mental health should seamlessly be assed at PHC level.

I was posted here as a psychiatric nurse, but I don't work as a psychiatric nurse, I rotate on all wards apart from maternity ward...I don't have enough time to talk to these patients so that they know more about mental illness (Psychiatric nurse HF 3).

The participants decried missed opportunity to provide mental health education to the patients they believed were in the communities and who may neither be aware about their mental illness nor the possibility of having it attended to. The PCPs attributed it to the lack of protected time to practice in the area of training as well as facilitation for mental health outreach activities.

...patients are in the community but they don't know about mental health concerns. They don't know that even mentally ill patients can be given treatment and improve to become important. I need to be supported go via the community and talk to the people; but that gap isn't there to go and talk to the people. Need to go to the churches and to different places and talk to the community (Psychiatric nurse HF 2).

Community outreach programs such as immunization are supported by the Ministry of Health; when asked why they did not utilize that opportunity to integrate mental health education, the participants' indicated that they would love to do it but because of limited time given and few personnel they are unable to include any other programs.

On the facilitative side, we found that some of the PCPs who used the UCG found them useful when screening for mental health problems. However, they alluded to challenges when it came to management of the patient after diagnosis.

The guidelines (UCG) are helpful because it is not something very hard to follow, you can reach the diagnosis easily... you face it when you do not have the drugs that you would have given the client and you opt for some simple drugs like diazepam instead of the real treatment (Nursing Officer HF 6).

\section{Motivation}

In this study, we found that some PCPs were not motivated to screen for mental illness using the UCG because they did not feel self-reliant. It could be attributed to limited knowledge and skills in both using the guidelines, and dealing with mental health problems.

I am not confident using the guidelines (UCG) because of the difficult terms in mental health... we were not taken through the guidelines to understand the terms and how to use them (Nursing Officer HF 5).

Some PCPs had not even seen the UCG at their respective health facilities. This probably was because of either the limited supply of hard copies or lack of knowledge that they were supposed to use the guidelines when assessing patients.

...personally, I have not seen those guidelines (UCG) in the room where I work from, I read them once when I was in Mbarara (Mbarara Regional Referral Hospital) like 3 years back (Enrolled Nurse HF 4).

When asked if screening for mental health problems caused any emotions, the PCPs response was to the affirmative although they indicated that it could not deter them from doing their job as healthcare providers. Emotions were mainly expressed by the female participants who felt distressed especially when dealing with children or unaccompanied persons. 
...I imagine like if this is my child in that condition, I feel like crying...I feel pity for the family. But again as a health worker of course we should not allow emotions to control us...I go ahead and see this client, I do not sit and cry with them...I try to counsel the patient until they are well (Nursing Officer HF 6).

Lack of trained mental health specialists at the health centers is another barrier we identified to the integration of mental health services into PHC. Some PCPs did not feel motivated to uptake the policy option because they did not have mental health specialists for immediate consultation. As remedy to that barrier, they proposed continuous medical education, and refresher courses in mental health to help them perform effectively.

...we do not have a psychiatric nurse to inquire about the difficult terms in mental health that we do not understand; we need CMEs or refresher courses (Midwife HF 4).

We found that integration of mental healthcare into routine care has been met with conflicting priorities thus limiting time for effective health education as expressed below.

I don't health educate mental health on a daily basis because there are other diseases to health educate in like diabetes and hypertension. But after getting those people from the general patients, I put them aside and talk about mental illness because that is my specialty where I talk much (Psychiatric nurse HF 3).

Integration of mental health services into PHC is a policy option in Uganda and most of the PCPs are aware that they are supposed to use the UCG when assessing for mental illness. However, there were no regulatory measures at the health facilities to encourage them screen for mental health problems.

There is nothing (measures) on ground to make us use the UCG. Maybe other units have but for us here we have not seen anything like that, not even in-service training in mental health. You are even the first person to come here in so many years on this mental health (Clinical Officer HF 6).

On the facilitative side, the PCPs were cognizant of the fact that if they followed the UCG, there would be improved care of the patients.

I think it will improve the care of people with the mental illness....we may not miss out on the cause and the management as well as the dosing

(Psychiatric nurse HF 3).
In addition, some participants indicated that they would feel contented with the service offered if they followed the UCG.

...if I know I have done what I am supposed to do confidently and I do it for real, I feel very good because I will have given a good quality of service (Nursing Officer HF 6).

When asked what kind of support was needed to enable them comfortably use the UCG when screening for mental health problems, one participant indicated that:

We need to be mentored on the assessment and management of people with mental illness so that it is easier for us when reading the guidelines (UCG) and managing or assessing for mental conditions (Nursing Officer HF 2).

\section{Recommendations from participants}

Notable recommendations from the study participants included the need to provide the PCPs with: a) copies of the UCG, b) summarized UCG for easier reference, c) in-service training for mental health, d) mental healthcare providers at each health center, and e) protected time for mental healthcare providers.

\section{Discussion}

In this study, we were interested in understanding the behavior of PCPs towards integration of mental health services into PHC. Cognizant of the fact that behaviors occur within a context of other behaviors, we found it necessary to identify the specific factors that affect the PCPs implementation of the policy option [35]. Procedurally, we used the COM-B framework to develop the interview guide and structure the analysis. The advantage of doing this was that we understood the factors affecting the PCPs implementation of mental health integration into $\mathrm{PHC}$, and identified the behavior (PCPs following the UCG when assessing for mental health problems) that needs to be addressed [33]. The COM-B framework has been used by various researchers, however, the closest study we found using the same approach was looking at barriers and facilitators to implementation of a web-based tool for diagnosis and monitoring of patients with depression [42].

Uganda is among the countries that undertook mental health reforms in conformity with a health policy philosophy which emphasizes decentralization of services to the lower administrative units. As a result, the UCG were developed with the aim to provide easy-to-use, practical and useful information on how to correctly diagnose and manage all common conditions [26] and avoid inappropriate 
variability in practice [43]. While clinical guidelines are systematically developed statements meant to assist practitioners in clinical decisions by providing cues to diagnosis and management of specific health conditions [1], we found in this study that most practitioners never found time to refer to the UCG. This was either due to lack of copies of the guidelines or conscious reluctance to refer to the guidelines [1].

\section{Barriers related to knowledge}

Our study confirms a knowledge gap among the PCPs in relation to mental disorders, the UCG for management of common disorders, and what they say about mental disorders at PHC. This result aligns with several studies [44-59] that speak about barriers related to the PCPs knowledge and skills in integration of mental health services into PHC; there is lack of belief that they are capable of adequately performing the recommendation [60].

\section{Barriers related to accessibility of the clinical guidelines} Concerning accessibility to the UCG, there was limited access whether by shared or personal copy because of the limited supply to the health centers; this was compounded by lack of sensitization about the guidelines at the point of introduction. There is need for sensitization of the PCPs to the UCG communicating the rationale for introducing evidence, and implementation of the guidelines [61, 62]; involvement of active efforts to raise awareness and promote interest in the UCG; as well as proactive efforts to understand the needs of the user and follow through to achieve a change in behavior [63].

The PCPs suggested that they would like a simplified format of the UCG in the form of a chart to which they could easily make reference. Thus, if usage of the UCG is to be promoted in rural Uganda, there is need to explore context specific alternatives to making the UCG better accessible to the intended users [62, 64]. Different audiences have different needs, learning styles and preferences, therefore when coming up with alternatives, there is need to be sensitive to the different segments or subgroups and come up with interventions that will maximally appeal to the targeted audience [64].

\section{Barriers related to point-of-care access}

The PCPs were faced with a point-of-care access challenge during the management of mental health problems; they did not have the opportunity to search for specific information at the point of the patient encounter when faced with a new condition because they did not have access to the UCG, thus reluctance to comply. In this study, point-of-care access is used to mean access to the UCG in whichever form including hard copy and softcopy.

Implementation studies show that the use of verbal prompts and visualization cues as engagement strategies promote uptake of the guidelines [65]. This is a potential strategy that could be utilized to improve integration of the mental health services into PHC in Uganda; verbal prompts such as regulatory measures to enforce adherence to the guidelines as well as visualization cues such as summarized guidelines either packaged in pocket size books or charts on the wall may go a long way in improving the point-of-care access.

Our study found that the rural health centers were good avenues for conducting research, and there were a number of donor funded projects with specific targets and various cues to help the PCPs attain the expected deliverables. This however was not the case for mental health; we found that mental health neither had a special program in the health centers nor specific targets to promote its screening and management at PHC level. As such, the PCPs felt that mental health was not among the priority areas because there was no support directed to it from anybody, thus directing their energies to where they are required to be accountable. Although it is good for health centers to be study sites, the limitation is that the health conditions that are not of interest to the funders tended to be neglected. The PCPs put more focus on health conditions that had set targets. In a study by Saraceno, van Ommeren [66], mental health investments in $\mathrm{PHC}$ are important but are unlikely to be sustained unless they are preceded by the development of community mental health services, to allow for training, supervision, and continuous support for primary care workers. In order to promote uptake of clinical guidelines, there is need for the policy makers to understand the local contexts of the health centers, and provide the relevant facilitation to allow for the local adaptation of guidelines $[67,68]$. In addition, allowing for local consensus is important to change social norms and, improve guideline implementation [69].

\section{Barriers related to integrating trained mental health providers}

Our study also found that PCPs trained as mental health providers and deployed at the various health centers were not entirely working in their area of training. They were not happy with the fact that they had to rotate to different wards providing general care as opposed to their area of interest (mental healthcare); this lowered their motivation to work. This however is a policy option that was adopted to address the issue of stigma [9].

When we analyzed the mental health integration policy, we found that: a) integration of mental health services into $P H C$ can be likened to task-shifting mental health into general care so that all clinicians regardless of whether they received specialized training in providing mental healthcare or not assess for mental health problems [9]. This policy may in part compromise the quality of care 
provided if the PCPs are provided with specialized training in mental healthcare. This is in agreement with a study by Jerene, Biru [70], who note that task-shifting mental health to general medical care requires more than brief provider training to enable proper care. b) if integration of mental health services into $\mathrm{PHC}$ is to be effective, there is need to provide co-training of the general PCPs with the mental health providers, and actively promote local collaborations (internal consultation) [70] otherwise, the policy of mental health integration defeats the purpose of specialized training of PCPs in mental health (psychiatry).

In spite of the numerous barriers, we found that the PCPs who utilized the UCG found them useful and believed that this would improve the care provided; this is in line with a study that was looking at implementing clinical guidelines in psychiatry [67].

\section{Limitations}

The results presented in our study are views from only clinical officers, nurses and midwives; we do not have the views of medial officers because they either did not consent to take part in the study or were not available at the time of the study. We therefore cannot rule out that they have divergent opinions.

Given that this was a case study of one district in one region of Uganda, we cannot confidently generalize the findings as applicable to other settings. There may be need for similar studies in the other regions of the country to confirm our findings.

\section{Conclusions}

Efforts to achieve successful integration of mental health services into PHC need to as much as possible fit into the context of the implementers. Much as the UCG spell out step-by-step procedure on how to utilize the guidelines for the management of common disorders, that is not good enough. There is need for a) adapting the guidelines into local context, this would involve sensitizing the users about the content and making them as accessible as possible, b) have some form of cues to enforce implementation of the policy option, and c) see how best to optimize the available expertize (mental health providers) in the integration process so that the trained mental health providers do not feel deprived of the opportunity to practice in their area of interest.

The findings of this study are important because they are an eye opener to the fact that policy options or guidelines should not be generalized but rather context specific to the areas where they are to be implemented, thus promoting better uptake.

\section{Additional file}

Additional file 1: Interview guide. (DOCX $17 \mathrm{~kb})$

\section{Abbreviations}

COM-B: Capability opportunity and motivation to behavior; GUREC: Gulu University Research Ethics Committee; HC: Health center; HF: Health facility; PCP: Primary care provider; PHC: Primary health care; Sida: Swedish International Development Cooperation Agency; UCG: Uganda clinical guidelines; UMHCP: Uganda minimum health care package; UNCST: Uganda National Council for Science and Technology; WHO: World Health Organization

\section{Acknowledgements}

The primary author (EW) is a PhD fellow under the Swedish International Development Cooperation Agency (Sida) and Makerere University Bilateral Program We recognize the great work done by Mariam Nakisekka and Christine Karungi our research assistants; this work would not been completed without your dedication to the data collection.

\section{Funding}

This study was funded by the "Swedish International Development Cooperation Agency (Sida) and Makerere University" where the lead author is a PhD student. The funding agency had no role in the design of the study or in the collection, analysis, interpretation of data and writing of the manuscript.

\section{Availability of data and materials}

Data on which this manuscript is based will not be publicly available since this work is still ongoing but will be available in future when the PhD program is completed.

\section{Authors' contributions}

EW developed the interview guide in consultation ESO, CO, and ZT; ESO interviewed the research assistants who were then trained by EW and CO; EW checked the transcripts before sharing them with ESO to generate codes; EW coded the data in consultation with ESO, CO, and ZT; analysis was performed by EW and content checked by psychiatrists SM and GZR. All authors read through the final manuscript and contributed to the final product. All authors read and approved the final manuscript.

\section{Authors' information}

EW is a Research Administrator/Manager and a PhD student in Implementation Science at Mbarara University of Science and Technology. $\mathrm{CO}$ is a senior researcher, a Pharmacologist, and Vice Chancellor of Mbarara University of Science and Technology.

GZR is a Psychiatrist and senior Lecturer in the Department of Psychiatry, Mbarara University of Science and Technology.

SM is an Associate Professor of Psychiatry in the Department of Psychiatry, Mbarara University of Science and Technology.

ZT is an Associate Professor of Medicine and of Health Policy, Department of Medical Education, California University of Science and Medicine.

ESO is a Social Anthropologist and Senior Lecturer at the Makerere University College of Health Sciences, Department of Psychiatry.

Ethics approval and consent to participate

Ethical approval was provided by the Gulu University Research Ethics Committee under application number 'GUREC 006/09/2017. The study was then registered with the Uganda National Council for Science and Technology under the number 'HS126ES'. All participants gave voluntary written informed consent to participate in this study.

\section{Consent for publication}

Not applicable.

\section{Competing interests}

The authors declare that they have no competing interests.

\section{Publisher's Note}

Springer Nature remains neutral with regard to jurisdictional claims in published maps and institutional affiliations.

\section{Author details}

'Department of Psychiatry, Mbarara University of Science and Technology, P.

O. Box 1410, Mbarara, Uganda. ${ }^{2}$ Department of Pharmacology and

Therapeutics and Vice Chancellor, Mbarara University of Science and 
Technology, Mbarara, Uganda. ${ }^{3}$ Department of Medical Education, California University of Science and Medicine, San Bernadino, California, USA. ${ }^{4}$ Mbarara University of Science \& Technology, Mbarara, Uganda. ${ }^{5}$ Department of Psychiatry, Makerere University College of Health Sciences, Kampala, Uganda.

\section{Received: 10 August 2018 Accepted: 5 November 2018}

\section{Published online: 26 November 2018}

\section{References}

1. Field E, Lohr K. Guidelines for clinical practice: from development to use. BMJ. 1993;306:17.

2. Grol R. Personal paper. Beliefs and evidence in changing clinical practice. BMJ. 1997;315(7105):418-21.

3. de Beurs DP, de Groot MH, de Keijser J, Verwey B, Mokkenstorm J, Twisk JW, et al. Improving the application of a practice guideline for the assessment and treatment of suicidal behavior by training the full staff of psychiatric departments via an e-learning supported train-the-trainer program: study protocol for a randomized controlled trial. Trials. 2013;14(1):9.

4. Grol R, Grimshaw J. From best evidence to best practice: effective implementation of change in patients' care. Lancet. 2003;362(9391):1225-30.

5. Kickbusch I. The contribution of the World Health Organization to a new public health and health promotion. Am J Public Health. 2003:93(3):383-8.

6. World Health Organization. Declaration of alma-ata: World Health Organization, Regional Office for Europe; 2004

7. Hall JJ, Taylor R. Health for all beyond 2000: the demise of the Alma-Ata declaration and primary health care in developing countries. Med J Aust. 2003;178(1):17-20.

8. World Health Organization. Report of the Director General on the work of WHO in 1978. p. 1979

9. World Health Organization. Mental Health Policiy, Planning \& Service Development Integrating Systems \& Services, Integrating People. Geneva: World Health Organization; 2007.

10. World Health Organization. In: Organization WH, editor. Improving health systems and services for mental health. Geneva: WHO Press; 2009.

11. World Health Organization. In: Organization WH, editor. The world health report 2008: primary health care now more than ever. Geneva: WHO Press; 2008

12. Barraclough F, Longman J, Barclay L. Integration in a nurse practitioner-led mental health service in rural Australia. Aust J Rural Health. 2016;24(2):144-50.

13. Bindman J, Johnson S, Wright S, Szmukler G, Bebbington P, Kuipers E, et al. Integration between primary and secondary services in the care of the severely mentally ill: patients' and general practitioners' views. Br J Psychiatry. 1997;171:169-74.

14. Chowdhury N. Integration between mental health-care providers and traditional spiritual healers: Contextualising Islam in the twenty-first century. J Relig Health. 2016;55(5):1665-71.

15. Coker $\mathrm{AO}$, Olugbile $\mathrm{OB}$, Oluwatayo O. Integration of mental healthcare into primary healthcare in Lagos, Nigeria: the way forward. Healthc Low-Resour Settings. 2015;3(2).

16. Bhana A, Petersen I, Baillie KL, Flisher AJ, The Mhapp Research Programme C. Implementing the World Health Report 2001 recommendations for integrating mental health into primary health care: a situation analysis of three African countries: Ghana, South Africa and Uganda. Int Rev Psychiatry. 2010;22(6):599-610.

17. McGough PM, Bauer AM, Collins L, Dugdale DC. Integrating behavioral health into primary care. Population health management. 2016;19(2):81-7.

18. Patel V, Maj M, Flisher AJ, Silva MJ, Koschorke M, Prince M, et al. Reducing the treatment gap for mental disorders: a WPA survey. World Psychiatry. 2010;9(3):169-76

19. Hailemariam M, Fekadu A, Selamu M, Medhin G, Prince M, Hanlon C Equitable access to integrated primary mental healthcare for people with severe mental disorders in Ethiopia: a formative study. Int J Equity Health. 2016;15(1):121

20. Kigozi F, Ssebunnya J, Kizza D, Cooper S, Ndyanabangi S. An overview of Uganda's mental health care system: results from an assessment using the world health organization's assessment instrument for mental health systems (WHO-AIMS). Int J Ment Heal Syst. 2010;4(1):1.

21. Mugisha J, Ssebunnya J, Kigozi FN. Towards understanding governance issues in integration of mental health into primary health care in Uganda. Int J Ment Heal Syst. 2016;10:25.
22. Ssebunnya J, Kigozi F, Kizza D, Ndyanabangi S. Integration of mental health into primary health care in a rural district in Uganda. Afr J Psychiatry. 2010; 13(2):128-31.

23. Kigozi F. Integrating mental health into primary health care-Uganda's experience. Afr J Psychiatry. 2007;10(1):17-9.

24. Amandua J, Bakeera S, Mpeka B, Sund B. Country case report Uganda. World Hosp Health Serv. 2002;39(3):24-5 8-30, 43, passim.

25. Ministry of Health. In: Health Mo, editor. Uganda Clinical Guidelines for Management of Common Conditions. Kampala: Rebublic of Uganda; 2012

26. Ministry of Health. Uganda Clinical Guidelines- National Guidelines for Management of Common Conditions; 2016.

27. Kakuma R, Minas H, van Ginneken N, Dal Poz MR, Desiraju K, Morris JE, et al. Human resources for mental health care: current situation and strategies for action. Lancet. 2011;378(9803):1654-63.

28. Ssanyu R. Mental illness and exclusion: putting mental health on the development agenda in Uganda. Policy brief 2. Kampala: Chronic Poverty Research Centre; 2007.

29. Fischer F, Lange K, Klose K, Greiner W, Kraemer A. Barriers and Strategies in Guideline Implementation-A Scoping Review. Healthcare (Basel). 2016:4(3):36.

30. Kitson A, Straus SE. The knowledge-to-action cycle: identifying the gaps Can Med Assoc J. 2010;182(2):E73-E7.

31. Hyett N, Kenny A, Dickson-Swift V. Methodology or method? A critical review of qualitative case study reports. Int I Qual Stud Health Well Being. 2014;9(1):23606

32. Baxter $\mathrm{P}$, Jack $\mathrm{S}$. Qualitative case study methodology: study design and implementation for novice researchers. Qual Rep. 2008;13(4):544-59.

33. Michie $S$, van Stralen MM, West R. The behaviour change wheel: a new method for characterising and designing behaviour change interventions. Implement Sci. 2011;6:42.

34. Michie S, Ashford S, Sniehotta FF, Dombrowski SU, Bishop A, French DP. A refined taxonomy of behaviour change techniques to help people change their physical activity and healthy eating behaviours: the CALO-RE taxonomy. Psychol Health. 2011;26(11):1479-98.

35. Michie S, Atkins L, West R. The behaviour change wheel: a guide to designing interventions. Needed: physician leaders; 2014. p. 26.

36. Catherine Houghton R, Dympna Casey R, David Shaw PhD C. Rigour in qualitative case-study research. Nurse Res. 2013;20(4):12.

37. Republic of Uganda. National Population and Housing Census 2014: Area Specific Profiles. UgandaBureauofStatistics; 2017.

38. Nsereko JR, Kizza D, Kigozi F, Ssebunnya J, Ndyanabangi S, Flisher AJ, et al. Stakeholder's perceptions of help-seeking behaviour among people with mental health problems in Uganda. Int J Ment Heal Syst. 2011;5(1):5.

39. Rebublic of Uganda. Health: Mbarara District Local Government; 2017. Available from: http://www.mbarara.go.ug/content/primary-and-earlychildhood-learning. cited 29 June 2018.

40. Braun V, Clarke V. Using thematic analysis in psychology. Qual Res Psychol. 2006;3(2):77-101.

41. Muhr T. Atlas. ti: qualitative data analysis, version 7. Berlin: Scientific Software Development $\mathrm{GmbH} ; 2013$.

42. Krog MD, Nielsen MG, Videbæk Le J, Bro F, Christensen KS, Mygind A. Barriers and facilitators to using a web-based tool for diagnosis and monitoring of patients with depression: a qualitative study among Danish general practitioners. BMC Health Serv Res. 2018;18:503.

43. Gundersen $\mathrm{L}$. The effect of clinical practice guidelines on variations in care. Ann Intern Med. 2000;133(4):317-8.

44. Abera M, Tesfaye M, Belachew T, Hanlon C. Perceived challenges and opportunities arising from integration of mental health into primary care: a cross-sectional survey of primary health care workers in south-west Ethiopia. BMC Health Serv Res. 2014;14:113.

45. Athie K, Menezes AL, da Silva AM, Campos M, Delgado PG, Fortes S, et al. Perceptions of health managers and professionals about mental health and primary care integration in Rio de Janeiro: a mixed methods study. BMC Health Serv Res. 2016;16(1):532.

46. Ayalon L, Karkabi K, Bleichman I, Fleischmann S, Goldfracht M. Barriers to the treatment of mental illness in primary care clinics in Israel. Adm Policy Ment Health Ment Health Serv Res. 2015.

47. Cowan J, Raja S, Naik A, Armstrong G. Knowledge and attitudes of doctors regarding the provision of mental health care in Doddaballapur Taluk, Bangalore rural district, Karnataka. Int J Ment Heal Syst. 2012;6(1):21.

48. Davis DW, Honaker SM, Jones VF, Williams PG, Stocker F, Martin E. Identification and management of behavioral/mental health problems in 
primary care pediatrics: perceived strengths, challenges, and new delivery models. Clin Pediatr. 2012;51(10):978-82.

49. Duffy M, Sharer M, Cornman H, Pearson J, Pitorak H, Fullem A. Integrating mental health and HIV Services in Zimbabwean Communities: a nurse and community-led approach to reach the Most vulnerable. J Assoc Nurses AIDS Care. 2017;28(2):186-98.

50. Fickel JJ, Parker LE, Yano EM, Kirchner JE. Primary care--mental health collaboration: an example of assessing usual practice and potential barriers. J Interprof Care. 2007;21(2):207-16.

51. Hill SK, Cantrell P, Edwards J, Dalton W. Factors influencing mental health screening and treatment among women in a rural south central Appalachian primary care clinic. J Rural Health. 2016;32(1):82-91.

52. Kapungwe A, Cooper S, Mayeya J, Mwanza J, Mwape L, Sikwese A, et al. Attitudes of primary health care providers towards people with mental illness: evidence from two districts in Zambia. Afr J Psychiatry. 2011;14(4):290-7.

53. Kigozi FN, Ssebunnya J. Integration of mental health into primary health care in Uganda: opportunities and challenges. Ment Health Fam Med. 2009; 6(1):37-42.

54. Knowles SE, Chew-Graham C, Adeyemi I, Coupe N, Coventry PA. Managing depression in people with multimorbidity: a qualitative evaluation of an integrated collaborative care model. BMC Fam Pract. 2015;16:32.

55. Martinez W, Galvan J, Saavedra N, Berenzon S. Barriers to Integrating Mental Health Services in Community-Based Primary Care Settings in Mexico City: A Qualitative Analysis. Psychiatr Serv. 2017;68(5):497-502.

56. Mesidor M, Gidugu V, Rogers ES, Kash-Macdonald VM, Boardman JB. A qualitative study: barriers and facilitators to health care access for individuals with psychiatric disabilities. Psychiatr Rehabil J. 2011;34(4):285-94.

57. Mosaku KS, Wallymahmed AH. Attitudes of primary care health workers towards mental health patients: a cross-sectional study in Osun state, Nigeria. Community Ment Health J. 2016.

58. Winer RA, Morris-Patterson A, Smart Y, Bijan I, Katz CL. Knowledge of and attitudes toward mental illness among primary care providers in Saint Vincent and the Grenadines. Psychiatry Q. 2013;84(3):395-406.

59. Zubkoff $L$, Shiner B, Watts BV. Staff perceptions of substance use disorder treatment in VA primary care-mental health integrated clinics. J Subst Abus Treat. 2016;70:44-9.

60. Lugtenberg M, Zegers-van Schaick JM, Westert GP, Burgers JS. Why don't physicians adhere to guideline recommendations in practice? An analysis of barriers among Dutch general practitioners. Implement Sci. 2009:4(1):54.

61. Forrest D, Hoskins A, Hussey R. Clinical guidelines and their implementation. Postgrad Med J. 1996;72(843):19-22.

62. Greenhalgh T. How to implement evidence based care. Oxford: Wiley Blackwell; 2018.

63. Lomas J. Diffusion, dissemination, and implementation: who should do what? Ann N Y Acad Sci. 1993;703(1):226-37.

64. Rogers EM. Diffusion of innovations: Simon and Schuster. New York: Free Press; 2010.

65. Slade SC, Hay-Smith J, Mastwyk S, Morris ME, Frawley H. Strategies to assist uptake of pelvic floor muscle training for people with urinary incontinence: a clinician viewpoint. Neurourol Urodyn. 2018.

66. Saraceno B, van Ommeren M, Batniji R, Cohen A, Gureje O, Mahoney J, et al. Barriers to improvement of mental health services in low-income and middle-income countries. Lancet. 2007;370(9593):1164-74.

67. Forsner T, Hansson J, Brommels M, Wistedt AA, Forsell Y. Implementing clinical guidelines in psychiatry: a qualitative study of perceived facilitators and barriers. BMC Psychiatry. 2010;10:8.

68. Harris MF, Lloyd J, Litt J, van Driel M, Mazza D, Russell G, et al. Preventive evidence into practice (PEP) study: implementation of guidelines to prevent primary vascular disease in general practice protocol for a cluster randomised controlled trial. Implement Sci. 2013;8:8.

69. Schectman JM, Schroth WS, Verme D, Voss JD. Randomized controlled tria of education and feedback for implementation of guidelines for acute low back pain. J Gen Intern Med. 2003;18(10):773-80.

70. Jerene D, Biru M, Teklu A, Rehman T, Ruff A, Wissow L. Factors promoting and inhibiting sustained impact of a mental health task-shifting program for HIV providers in Ethiopia. Glob Ment Health. 2017;4:e24.

\section{Ready to submit your research? Choose BMC and benefit from:}

- fast, convenient online submission

- thorough peer review by experienced researchers in your field

- rapid publication on acceptance

- support for research data, including large and complex data types

- gold Open Access which fosters wider collaboration and increased citations

- maximum visibility for your research: over $100 \mathrm{M}$ website views per year

At BMC, research is always in progress.

Learn more biomedcentral.com/submissions 